\title{
Influence of dissipation on a low-voltage dc current in a long SNS junction
}

\author{
Sergey I. Kulinich ${ }^{1,2}$ and Robert I. Shekhter ${ }^{1}$ \\ ${ }^{1}$ Department of Applied Physics, Chalmers University of Technology and Göteborg University, \\ SE-412 96 Göteborg, Sweden \\ E-mail: shekhter@fy.chalmers.se
}

${ }^{2}$ B. Verkin Institute for Low Temperature Physics and Engineering of the National Academy of Sciences of Ukraine, 47 Lenin Ave., Kharkov 61103, Ukraine

Received April 19, 2002

\begin{abstract}
The dc current through a voltage-biased long transparent SNS junction in a dissipative regime is considered. The problem under certain conditions is mapped onto exactly solvable model of energy pumping into a quasiballistic $1 \mathrm{D}$ quantum ring driven by time-dependent magnetic flux. A rich peak-like structure of the subgap current at low voltages is predicted. The maxima in the current correspond to resonant energy absorption for fractional values of the normalized bias voltage.
\end{abstract}

PACS: 74.80.Fp, 74.50.+r

\section{Introduction}

The nonequilibrium properties of mesoscopic conducting systems differ significantly from those in bulk materials. The reason is the dominant role of quantum coherent dynamics which develops on a time scale, shorter than the relaxation times in the system. As a result, quantum interference phenomena crucially affect the nonlinear kinetics of mesoscopic conductors (see, e.g., [1]).

Nonsuperconducting mesoscopic structures have been shown to be extremely sensitive to external electromagnetic field, producing giant mesoscopic fluctuations of the conductance [2]. Mesoscopic rings placed in a time-dependent magnetic flux are characterized by strong resonant selection in the microwave power absorbed (fractional pumping of mesoscopic rings [3]). The origin of all of the above effects is quantum interference between different paths of dynamical evolution of electrons driven by external fields.

Superconducting nanostructures in which quantum coherence is a «macroscopic» feature of large numbers of electrons should reveal nonequilibrium coherent dynamics in an even more pronounced way. Energy dissipation in mesoscopic supercon- ducting weak links is an example of phase coherence nonequilibrium phenomenon in superconducting mesoscopics.

In the present paper we will consider the effect of fractional dissipation of electric power in voltage-biased long ballistic SNS structures. Andreev

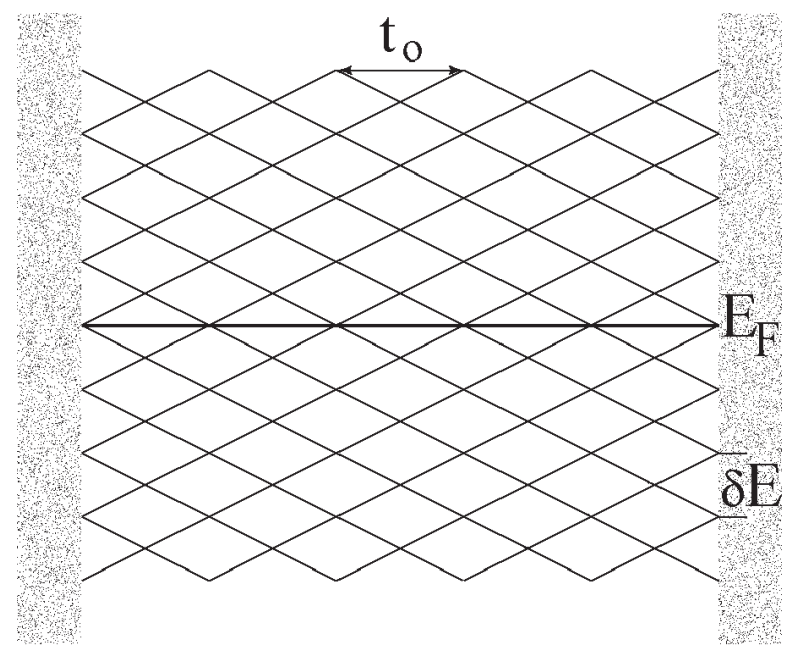

Fig. 1. The set of Andreev levels, $E_{n}^{ \pm}$, as a function of time $t$ in a long ballistic SNS junction. $\delta E$ is a level spacing, $t_{0}$ is a «quasiperiod» in the problem. 
levels representing the energy spectrum in a long SNS transparent junction are shown in Fig. 1. If a small voltage $e V<<\hbar v_{F} / L$ is applied $\left(v_{F}\right.$ is the Fermi velocity, $L$ is the junction length), the quantum evolution of Andreev states can be represented as a motion along the lines of Fig. 1. which is interrupted by scattering events in the vicinity of the crossing points. This scattering splits the trajectory and results in the appearance of a number of alternative quantum paths for the displacement of the system in energy space. The interference of such paths ends at the moment of time when relaxation stops the dynamical pumping of the system.

Two different scenario of dynamical evolution of quasiparticles in a normal region are possible depending on the intensity of energy relaxation. In the weak dissipation limit the dynamical drift along the energy lines eventually brings the system out of the energy gap. The dissipation of energy takes place in the leads (bulk superconductors) far from the junction. This scenario corresponds to the now traditional picture of current formation by multiple Andreev reflections (MAR) [4-6]. In contrast, in the strong dissipation limit (which is effectively always the case at $V \rightarrow 0$ ) the coherent dynamical evolution is interrupted by relaxation, preventing quasiparticles from leaving the gap region. This is the case where power emission is localized within a weak link and the formation of current is totally due to quantized Andreev states. This is the case we will consider below.

The interference pattern for energy absorption will be sensitive to commensurability between the typical time of interscattering dynamics $t_{0} \sim \hbar / e V$ and the periods $t_{A} \sim \hbar / \delta E$ ( $\delta E$ is the level spacing) characterizing the dynamics of quantized Andreev states. Since the absorbed power $W$ directly determines the average current $I$ in the system, the above interference should produce nonmonotonic $I-V$ characteristics with the peaks corresponding to maxima of the resonant absorption, $t_{0} / t_{A}=p / q(p, q$ are integers $)$. The calculation of «fractional structure» of $I-V$ characteristics is the subject of the present work.

\section{The average absorption energy}

We begin with the description of a set of bound states in a single-mode SNS junction. The energies of Andreev levels, $E_{n}^{\sigma}$, as a function of the phase difference $\varphi=\varphi_{R}-\varphi_{L}$ between the two superconductors are found from the transcendental equation [7]

$$
\frac{E_{n}^{(\sigma)}}{\delta E}-\sigma \frac{\varphi}{2 \pi}=n+\frac{1}{\pi} \arccos \frac{E_{n}^{(\sigma)}}{\Delta},
$$

where $\delta E=\pi \hbar v_{F} / L$ is the level spacing, $n=0, \pm 1, \pm 2, \ldots$, and $\sigma= \pm 1$. In a long $\left(L>>\xi_{0}, \xi_{0}\right.$ is the coherence length) junction the spectrum Eq.(1) for $E<<\Delta$ ( $\Delta$ is the modulus of the order parameter) is reduced to a set of equidistant levels that depend linearly on $\varphi$ :

$$
\frac{E_{n}^{( \pm)}}{\delta E}=n+\frac{\pi \pm \varphi}{2 \pi} .
$$

The terms cross at points $\varphi_{p}=\pi p$ ( $p$ is the integer). This degeneracy is lifted in the presence of normal scattering in the junction. We will model the normal scattering by a weak point-like impurity ( $U_{0}<<\delta E, U_{0}$ is the impurity potential) placed at one of the interfaces (asymmetric junction). In this case the gaps $\Delta_{n}$ that are opened at the degeneracy points are small and $n$-independent, $\Delta_{n} \simeq U_{0}$.

For a voltage-biased junction the phase difference $\varphi$ according to the Josephson relation ( $\dot{\varphi}=2 e \mathrm{~V} / \hbar)$ depends linearly on time, and the problem becomes nonstationary and nonequilibrium. It can be reformulated as a transport problem in energy space, where in the general situation both the bound $(E<\Delta)$ and the scattering $(E>\Delta)$ states contribute to the dc current. We will consider a long ballistic SNS junction in a dissipative regime, that is, in the case when dissipation influences the dynamics of quasiparticles in the normal region. In the presence of dissipation the maximum energy gained by quasiparticles in the normal region can be much smaller than $\Delta$. In this case only the bound states in a small strip around $E_{F}$ are involved in dynamical processes, and their spectrum can be approximated as a linear one, Eq.(2). Thus, our problem is reduced to a transport problem on a set of equidistant Andreev levels (see Fig. 1).

It is evident that for a not fully transparent junction the dc current will be zero if one neglects the interlevel tunneling. So, to get finite current we have to consider electron transitions between the levels. If the ballistic time $t_{0} / 2$ of electron motion between the transition points, $t_{0}=\pi \hbar / \mathrm{eV}$, is much larger than the characteristic scattering time $t_{s} \sim \hbar / U_{0}$, the interlevel transitions will take place mostly in the vicinity of degeneracy points, where the interlevel spacing is the smallest $\left(\sim U_{0}\right)$. Therefore, at low voltages $e V<<U_{0}<<\delta E$ the dynamics of quasiparticles in energy space on a set of Andreev levels can be represented as a free motion between the «transition points» $\varphi_{p}=\pi p$ and a scattering at these points described by a unitary 
$2 \times 2$-matrix $\hat{S}$. It is convenient to parametrize the scattering matrix by the amplitude $\tau$ of interlevel transition:

$$
\hat{S}=\left(\begin{array}{cc}
\sqrt{1-\tau^{2}} & i \tau \\
i \tau & \sqrt{1-\tau^{2}}
\end{array}\right)
$$

At low voltages the electron transition amplitude is described by the Landau-Zener quasiclassical formula (see, e.g., Ref.8)

$$
\tau=\exp \left(-\frac{\pi^{2}}{2} \frac{U_{0}^{2}}{\delta E e V}\right)
$$

The dc current $I$ in a dissipative junction can be expressed in terms of the average energy accumulation rate $W$ by the standard formula $I=W / V$. To calculate this quantity we consider the energy absorption rate averaged over the time interval $\left[-t_{0} / 2, t_{0} / 2\right]$,

$$
W(t)=\frac{1}{t_{0}} \int_{t_{-}}^{t_{+}} d t^{\prime} \operatorname{Tr}\left\{\rho\left(t^{\prime}\right) \dot{\mathcal{H}}\left(t^{\prime}\right)\right\},
$$

where $t_{ \pm}=t \pm t_{0} / 2, \dot{\mathcal{H}} \equiv \partial_{t} \mathcal{H}(t)$. The single-electron density matrix is calculated from the equation

$$
\frac{\partial \rho}{\partial t}=\frac{i}{\hbar}[\rho, \mathcal{H}]-v\left(\rho-f_{0}\right),
$$

where $v$ is the relaxation rate and $f_{0} \equiv f_{0}[\mathcal{H}]$ is the equilibrium Fermi-Dirac distribution corresponding to the Hamiltonian

$$
\begin{aligned}
& \mathcal{H}=i \hbar v_{F} \sigma_{3} \tau_{3} \frac{\partial}{\partial x}+ \\
& +\Delta(x)\left\{\sigma_{1} \cos \varphi / 2-\operatorname{sgn} x \sigma_{2} \sin \varphi / 2\right\} .
\end{aligned}
$$

In expression (4) $\sigma_{i}, \tau_{i}$ are the Pauli matrices in electron-hole and «left-right» spaces, respectively, and $\Delta(x)=\Delta \Theta(|x|-L / 2) \quad$ (here $\Theta(x)$ is the Heaviside step function).

The solution of Eq.(3) can be expressed in terms of the evolution operator $u\left(t, t^{\prime}\right)$ :

$$
\rho=v \int_{-\infty}^{t} d t^{\prime} \mathrm{e}^{v\left(t^{\prime}-t\right)} u\left(t, t^{\prime}\right) f_{0}\left\{\mathcal{H}\left(t^{\prime}\right)\right\} u^{+}\left(t, t^{\prime}\right) .
$$

Then for the energy absorption rate one gets

$$
W(t)=\frac{1}{t_{0}} \int_{t_{-}}^{t_{+}} d t^{\prime} \mathrm{e}^{-v t^{\prime}} H\left(t^{\prime}\right) \int_{-\infty}^{t^{\prime}} d t^{\prime \prime} \mathrm{e}^{v t^{\prime \prime}} F\left(t^{\prime \prime}\right)
$$

where

$$
\begin{gathered}
H(t)=u^{+}(t, 0) \dot{\mathcal{H}}(t) u(t, 0), \\
F(t)=u^{+}(t, 0) \dot{f}_{0}\{\mathcal{H}(t)\} u(t, 0) .
\end{gathered}
$$

With the help of the periodic symmetry property of the Hamiltonian (4),

$$
H\left(t+t_{0}\right)=\sigma_{3} H(t) \sigma_{3},
$$

it is easy to show that the average power absorption does not depend on time, $W(t)=W=$ const, and without any loss of generality we can put $t=0$ in Eq.(5). It is convenient to divide the whole time interval of integration $\left(-\infty, t^{\prime}\right)$ in Eq. (5) into discrete intervals $\left[-t_{0} / 2-m t_{0} ; t_{0} / 2-m t_{0}\right], m=$ $=0,1, \ldots$. Finally the formula for the average energy absorption rate takes the form

$$
W=\frac{1}{t_{0}} \operatorname{Tr} \sum_{m=0}^{\infty} \mathrm{e}^{-2 \tilde{v} m} H P^{m} F P^{-m}+Q,
$$

where $\tilde{v}=\frac{1}{2} v t_{0}, P=\sigma_{3} u\left(t_{0}, 0\right)$ and

$$
\begin{gathered}
H=\int_{-t_{0} / 2}^{t_{0} / 2} d t \mathrm{e}^{-v t} H(t), F=\int_{-t_{0} / 2}^{t_{0} / 2} d t \mathrm{e}^{-v t} F(t), \\
Q=\frac{1}{t_{0}} \operatorname{Tr} \int_{-t_{0} / 2}^{t_{0} / 2} d t^{\prime} \mathrm{e}^{-v t^{\prime}} H\left(t^{\prime}\right) \int_{-t_{0} / 2}^{t^{\prime}} d t^{\prime \prime} \mathrm{e}^{v t^{\prime \prime}} F\left(t^{\prime \prime}\right) .
\end{gathered}
$$

In what follows we will consider the most interesting case of moderately weak dissipation $\delta E / \Delta<<\tilde{v}<<1$.

As one can see from Eq.(6), the only operator associated with a long-time dynamics of our system is the unitary operator $P$. This means that the $I-V$ characteristics of a long dissipative SNS junction at low bias voltage and for a weak dissipation are determined by a spectral properties of $P: P|\beta\rangle=\mathrm{e}^{i \beta}|\beta\rangle$. In the basis of Andreev levels $|n, \pm\rangle$ this operator coincides with the one studied in considerable detail in Ref. 3 for the problem of energy pumping into a quasiballistic quantum ring. It was shown in Ref. 3 that the spectrum of $P$ crucially depends on the number properties of the quantity $\alpha=\{\delta E / 4 e V\}$ (here $\{x\}$ stands for the fractional part of $x$ ).

For an irrational $\alpha$ the spectrum of $P$ is discrete and all eigenfunctions are localized. In the limit $v \rightarrow 0$ the average energy absorption rate can be estimated as

$$
W_{i}=v \delta E R_{\text {loc }}^{2},
$$

where $R_{\text {loc }}$ is the dimensionless localization radius [3]. An analytical expression for $R_{\text {loc }}$ was derived in our previous paper: 


\section{Conclusion}

$$
R_{\mathrm{loc}}^{2}=\sum_{l=0}^{\infty} \frac{\tau^{2 l}}{\sin \pi \alpha l} .
$$

From Eqs.(7),(8) one can see that for «irrational case» the absorption power tends to zero in the limit $v \rightarrow 0$.

For rational values $\alpha=p / q(p<q$ are integers $)$ the eigenfunctions of $P$ are the Bloch functions [3]. In this case the absorbed power in the limit $v \rightarrow 0$ takes the form

$$
W_{r}=\frac{\delta E}{v}\left(\frac{e V}{\pi \hbar}\right)^{2}\left(1-\sqrt{1-\tau^{2 q}}\right)+O(v),
$$

(in the last expression we assume that $p \neq 0$; if $p=0$ we must put $q=1$ in Eq.(9)).

To compare the contributions (7) and (9) to the $I-V$ curves, one first of all needs to find the relevant energy scale to satisfy $W_{r} \gg W_{i}$. It is easy to show that the above inequality will hold (for not-too-big values of $q$ ) when the bias voltage falls in the interval $\hbar v<<e V<<U_{0}$ and for $e V \sim \pi U_{0}^{2} / \delta E$ when the amplitude $\tau$ of interlevel transitions is not exponentially small. At these voltages the $I-V$ characteristics of a long transparent SNS junction should demonstrate specific (peak-like) structure (see Fig. 2) arising from the effect of fractional pumping of energy into the quasiballistic electron system with equidistant quantized energy levels.

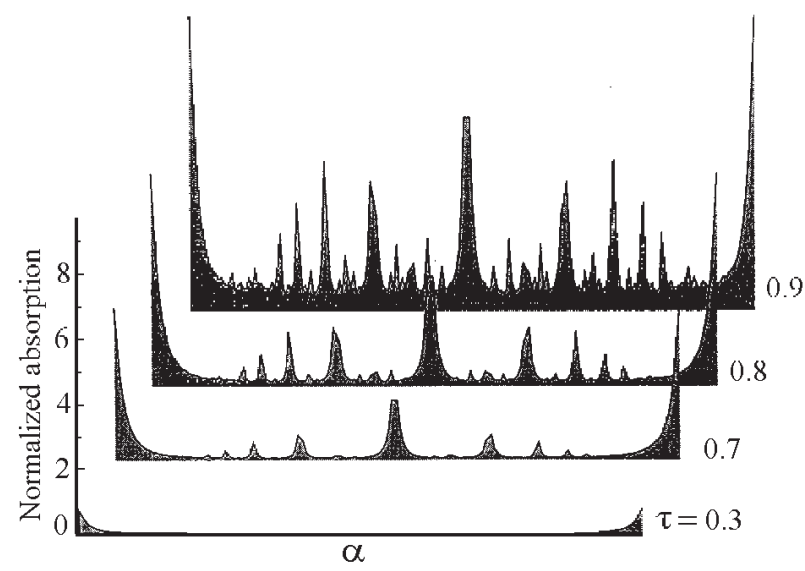

Fig.2. The normalized absorption energy, $W / W_{0}$, as a function of parameter $\alpha, 0<\alpha<1$, for different Landau-Zener tunneling amplitudes; $W_{0}=\delta E / t_{0}$. The value $\tilde{v}=0.05$ for the dimensionless relaxation rate was used.
In recent experiments $[9,10]$ the effects of quantum coherence were observed in a dissipative long SNS junction irradiated by an electromagnetic field. These experiments clearly demonstrated that dissipation in a long transparent SNS junction is unable to wash out quantum interference effects produced by the coherent dynamics of quasiparticles in the normal region. The orthodox theory [4-6] of subgap structures in $I-V$ characteristics of SNS junction is based on the Landauer-Buttiker approach, where it is assumed that dissipation occurs only in the leads (bulk superconductors) and does not influence the dynamics of electrons inside the normal region. Recently [11] a theory of MAR was applied for the description of subgap structures in long $\left(L>>\xi_{0}\right)$ quasiballistic junctions (see also Refs.12,13). It is clear that for a real long SNS junction at sufficiently low bias voltages the dissipation will take place in the normal region, and the junction should be treated as a dissipative one.

We have proposed an approach in which both the coherent dynamics of quasiparticles in a long junction and the relaxation effects inside the normal region determine the current through a weak link.

We showed that the problem under certain conditions can be mapped onto an exactly solvable model of energy dissipation in a quasiballistic quantum ring driven by a linearly time-dependent magnetic flux. The existence of this mapping can be understood physically from the fact that the sets of energy levels of the two problems under consideration are identical.

We derived analytical expressions for the dc current through the junction at low bias voltages in the limit of moderately weak dissipation $e V(\delta E / \Delta)<<\hbar v<<\delta E$ and predicted nontrivial (peak-like) subgap structure of $I-V$ characteristic at low voltages $\hbar v<<e V<<\delta E$. The peaks in the current correspond to maxima of absorbed power and they are a manifestation of the effect of fractional pumping of energy into a quasiballistic electron system with an equidistant set of energye levels.

The authors thank L.Y.Gorelik, M. Jonson, and I.V. Krive for valuable discussions. Financial support from the Swedish NFR (S.K.,R.S.) and the U. S. Department of Energy Office of Science through contract No. W-31-109-ENG-38 (R.S.) is gratefully acknowledged. 
1. L. Y. Gorelik, M. Jonson, R. I. Shekhter, and O. Tageman. In: Proc of the NATO Advanced Study Institute on Frontiers in Nanoscale Science of Micron/Submicron Devices, Kiev, August 1995, NATO ASI Series E: Applied Sciences 328 (1996) p. 225, A-P.Jauho and E.V.Buzaneva (eds.).

2. L. Y. Gorelik, A. Grincwajg, V. Z. Kleiner, R. I. Shekhter, and M. Jonson, Phys. Rev. Lett. 73, 2260 (1994); L. Y. Gorelik, F. Maao, R. I. Shekhter, and M. Jonson, Phys. Rev. Lett. 78, 3169 (1997).

3. L. Gorelik, S. Kulinich, Yu. Galperin, R. I. Shekhter, and M. Jonson, Phys. Rev. Lett. 78, 2196 (1997); Fiz. Nizk. Temp. 24, 1181 (1998) [Low Temp. Phys. 24, 887 (1998)].

4. G. E. Blonder, M. Tinkham, and T. M. Klapwijk, Phys. Rev. B25, 4515 (1982).

5. E. N. Bratus', V. S. Shumeiko, and G. Wendin, Phys. Rev. Lett. 74, 2110 (1995).
6. D. Averin and A. Bardas, Phys. Rev. Lett. 74, 1831 (1995).

7. I. O. Kulik, Sov.Phys. JETP 57, 1745 (1969).

8. L. D. Landau, Phys. Z. Sov. 2, 46 (1932); C. Zener, Proc. R. Soc. London A137, 696 (1932)

9. P. Dubos, H. Courtois, O.Buisson, and B.Pannetier, Phys. Rev. Lett. 87, 206801 (2001).

10. P. Dubos, H. Courtois, B. Pannetier, F. K. Wilhelm, A. D. Zaikin, and G. Schon, Phys. Rev. B63, 064502 (2001).

11. A. Ingerman, G. Johansson, V. S. Shumeiko, and G. Wendin, Phys. Rev. B64, 1445 (2001).

12. U. Gunsenheimer and A. D. Zaikin, Phys. Rev. B50, 6317 (1994).

13. M. Hurd, S. Datta, and P. F. Bagwell, Phys. Rev. B54, 6557 (1996). 